\title{
Surface Chemistry Enhancements for the Tunable Super-Liquid Repellency of Low-Surface-Tension Liquids
}

\author{
William S. Y. Wong*(1) \\ Nanotechnology Research Laboratory, Research School of Engineering, The Australian National University, Canberra ACT 2601, \\ Australia \\ Max Planck Institute for Polymer Research, Ackermannweg 10, D-55128 Mainz, Germany
}

Supporting Information
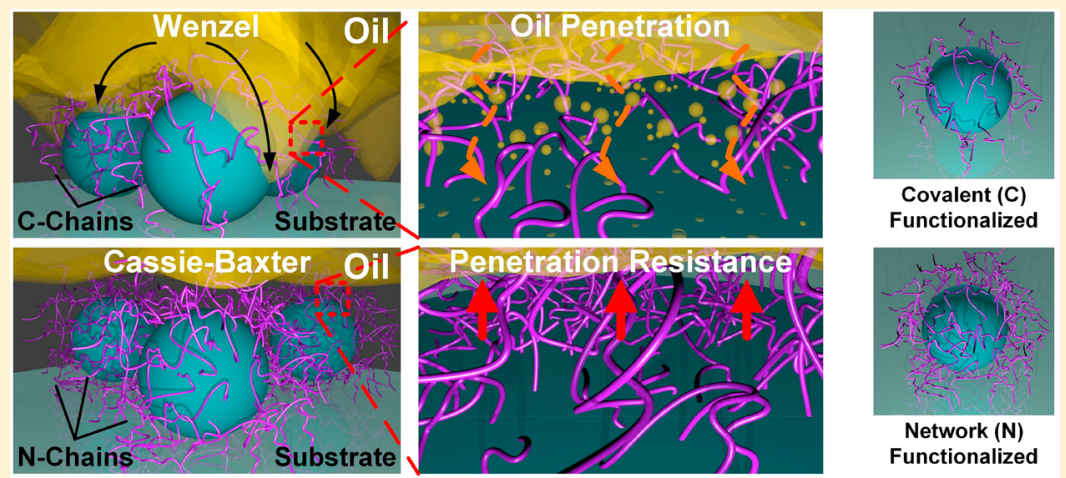

ABSTRACT: Super-hydrophobic, super-oleo(amphi)phobic, and super-omniphobic materials are universally important in the fields of science and engineering. Despite rapid advancements, gaps of understanding still exist between each distinctive wetting state. The transition of super-hydrophobicity to super-(oleo-, amphi-, and omni-)phobicity typically requires the use of reentrant features. Today, re-entrant geometry induced super-(amphi- and omni-)phobicity is well-supported by both experiments and theory. However, owing to geometrical complexities, the concept of re-entrant geometry forms a dogma that limits the industrial progress of these unique states of wettability. Moreover, a key fundamental question remains unanswered: are extreme surface chemistry enhancements able to influence super-liquid repellency? Here, this was rigorously tested via an alternative pathway that does not require explicit designer re-entrant features. Highly controllable and tunable vertical network polymerization and functionalization were used to achieve fluoroalkyl densification on nanoparticles. For the first time, relative fluoro-functionalization densities are quantitatively tuned and correlated to super-liquid repellency performance. Step-wise tunable super-amphiphobic nanoparticle films with a Cassie-Baxter state (contact angle of $>150^{\circ}$ and sliding angle of $<10^{\circ}$ ) against various liquids is demonstrated. This was tested down to very low surface tension liquids to a minimum of ca. $23.8 \mathrm{mN} /$ $\mathrm{m}$. Such findings could eventually lead to the future development of super-(amphi)omniphobic materials that transcend the sole use of re-entrant geometry.

KEYWORDS: Super-hydrophobicity, super-oleophobicity, super-amphiphobicity, super-omniphobicity, liquid repellency, surface energy density

\section{INTRODUCTION}

Super-lyophobic ("solvent-fearing") materials ${ }^{1}$ have immense influence over a range of commercial, industrial, and research sectors. Their unique interactions with liquids have warranted applications in the fields of self-cleaning coatings, ${ }^{2-7}$ (micro)fluidics, ${ }^{8-11}$ intelligent liquid management systems, ${ }^{12-16}$ and even smart stimuli-responsive materials. ${ }^{17-19}$ In recent years, expanding beyond super-hydrophobicity, ${ }^{20,21}$ two new classes of super-lyphobicity have been gaining immense research momentum. These are outlined by the states of superoleo(amphi)phobicity ${ }^{22,23}$ and super-omniphobicity. ${ }^{23,24}$ Despite their superior performance against ultra-low surface tension liquids, they can be notoriously difficult to fabricate, owing to the need for geometrically re-entrant textures. ${ }^{24-26}$
Moreover, owing to the inherently unstable re-entrant profile, they may also suffer from mechanical stability. ${ }^{22,27-29}$ The poor mechanical stability of purely re-entrant geometries is further limited by the mode of synthesis. Methods such as lithography and chemical vapor deposition derived soot nanoparticles ${ }^{22}$ or nanofilaments ${ }^{30}$ cannot be easily combined into a binder-based system ${ }^{5,6,31}$ for the eventual enhancement of robustness. The concept of re-entrant geometry is wellsupported by both theoretical modeling and experimental validations. ${ }^{26,29,32,33}$ State-of-the-art methods primarily attrib-

Received: December 12, 2018

Revised: January 20, 2019

Published: February 6, 2019 
ute the effect of super-amphiphobicity to the presence of reentrant geometry. This is a well-known route. For instance, they describe inherently re-entrant nanofilaments, ${ }^{30}$ re-entrant nanoparticle agglomerates, ${ }^{22,27}$ or overhanging geometries in $\mathrm{TiO}_{2}-\mathrm{SWNT}^{34}$ composites. In addition, soot-particle coatings take up to four distinct steps and require skilled precision, chemical vapor deposition, and over a period of at least 3 days to produce. $^{22}$ Nanofilament coatings also employ the use of chemical vapor deposition or liquid-immersion growth under precisely controlled conditions. ${ }^{30}$ Both methods are still comparatively complicated to be readily adopted by the industry. Evidently, this dogma can also be potentially debilitating toward future research progress and industrial development of the field.

Wettability is a surface phenomenon that relies on two factors: namely, the surface energy and surface topography. ${ }^{16,35}$ Today, super-(oleo-, amphi-, and omni-)phobicity typically exploit the latter. ${ }^{26,29,32,33}$ Research into the former is often bracketed by the conceptual minimum surface energy of a $-\mathrm{CF}_{3}$ group, ${ }^{36}$ which is often used to justify all superlyophobic states, ranging from super-hydro- to super-(oleo-, amphi-, and omni-)phobicity. ${ }^{23,37,38}$ As of the time of writing, no literature currently exists on the configurational optimization of fluoroalkyl moieties for super liquid repellency or superamphi(omni)phobicity. The grafting density of fluoroalkyl groups with nanosilica was previously investigated but only for relative (super)hydrophobicity. ${ }^{39}$ Moreover, functionalization density remained fairly low (ca. $26.9 \% \mathrm{w} / \mathrm{w})^{39}$ compared to some of the most heavily fluorinated polyhedral oligomeric silsesquioxane (F-POSS) materials known, ${ }^{40}$ reaching a staggering organic content of $92 \% \mathrm{w} / \mathrm{w}$. Notably, F-POSS is also coincidentally found in many works that describe facile, stochastic development of super-(oleo-, amphi- and omni)phobic states. ${ }^{41-43}$

In this work, we revisit an old conundrum, focusing on the potentially tunable and maximum extent of surface fluorofunctionalization. This is investigated specifically with respect to its corresponding implications on wetting. The central theme of this work investigates the potential of increasing fluoro-functionalization for achieving different states of superliquid repellency. We explore this by using a multilayered, selfcondensing, network-polymerization-based functionalization procedure. McCarthy et al. once described this mechanism as a potential side-reaction by trichlorosilanes, giving rise to vertical polymerization and a cross-linked $3 \mathrm{D}$ siloxane backbone. ${ }^{44}$ Here, we utilize an extension of this concept: supersaturation functionalization, drawing an analogy to the state of a medium that is saturated with more material than normal. The grafting density demonstrated here reached ca. $53.5 \pm 1.6 \% \mathrm{w} / \mathrm{w}$ while showcasing distinctive step-wise tunability in liquid repellent behaviors. These occurred at $19.7 \%, 30.8 \%, 44.3 \%$, and $53.6 \% \mathrm{w} / \mathrm{w}$ toward water, ethylene glycol, hexadecane, and $n$-decane $(72.8,47.7,27.4$, and 23.8 $\mathrm{mN} / \mathrm{m}$ ), respectively. This limit plateaus at ca. $50-60 \% \mathrm{w} / \mathrm{w}$. For the first time, we are able to quantify and correlate the relative density of functional groups to super liquid repellency performance.

Results and Discussion. Supersaturation Functionalization and Covalent-Oligomeric Network Systems. In contrast to previous studies, ${ }^{27,39}$ spontaneous but conventionally undesirable $e^{44,45}$ self-condensation of chlorosilanes is allowed to take place under the chosen reaction environment (see the experimental details in the Supporting Information). These conditions led to stochastic polymerization reactions on the hydroxyl groups (on fumed silica), resulting in a combination of covalent bonding and oligomeric functionalization. ${ }^{44}$ Confirmation of covalent bonding and network polymerization was verified by higher-than-normal surface grafting density (4 $\mu \mathrm{mol} \cdot \mathrm{m}^{-2}$ ) and thermal stability of functionalized silanes under thermogravimetric analysis (TGA). These coupled reactions (Figure S1) enabled the formation of a network, resulting in the supersaturation functionalization of a typical amorphous silica interface, beyond the limitations of the monolayer-based model (Figure 1a). Initially, low grafting ratios result in more

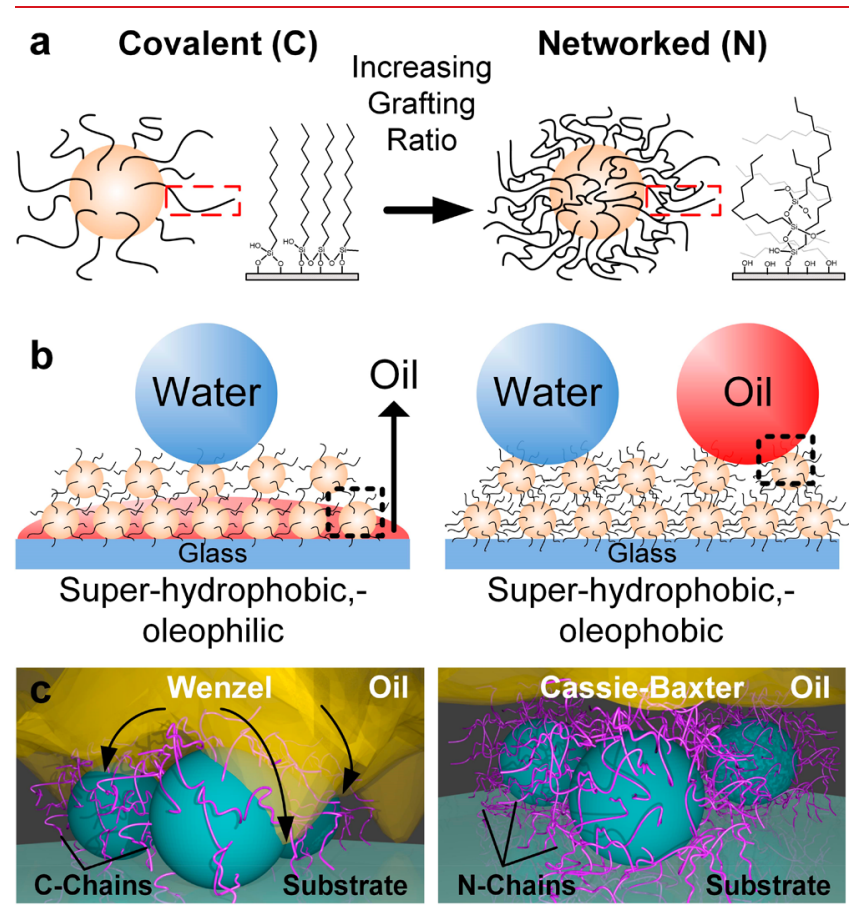

Figure 1. Re-examining the roots of super liquid repellency: surfaceenhanced dewettability. (a) Increase in grafting density via selfcondensation functionalization, leading to the network formation of fluoro-silica shells around nanoparticles. Covalent: near-stoichiometric but low-density attachment of fluoroalkylsilanes. Network: stochastic but high-density poly-formation of fluoro-silica networks. $\mathrm{F}$ and $\mathrm{H}$ atoms are not illustrated for conciseness. Low (covalent) and high (network) grafting density based nanoparticulate surfaces exhibiting tunable super liquid repellency. (b) Equivalent CassieBaxter-based super-hydrophobic-to-superamphiphobic properties with surface chemistry-tunable enhancements even against lowsurface-tension (left to right). Magnification of the local interfaces as proposed in panel c. (c) Schematic proposing the use of densified fluoroalkyl layers (right) vs thin fluoroalkyl layers (left) in preventing contact line advancement at the nanoscopic level by low-surfacetension oils.

covalent-monolayer formation due to the lack of steric hindrance. This is showcased by normal surface grafting densities $\left(1-2 \mu \mathrm{mol} \cdot \mathrm{m}^{-2}\right)$. Later, higher grafting ratios resulted in the inevitable enhancement of surface grafting density through oligomeric network polymerization. This higher-thannormal surface grafting density $\left(4 \mu \mathrm{mol} \cdot \mathrm{m}^{-2}\right)$ is indicative of a thickened functional layer that builds upon the original layers, which must be induced by network polymerization (Figures 3 and 4). This mechanism was once proposed by McCarthy et al. as a potential side-reaction of trichlorosilanes, resulting in 

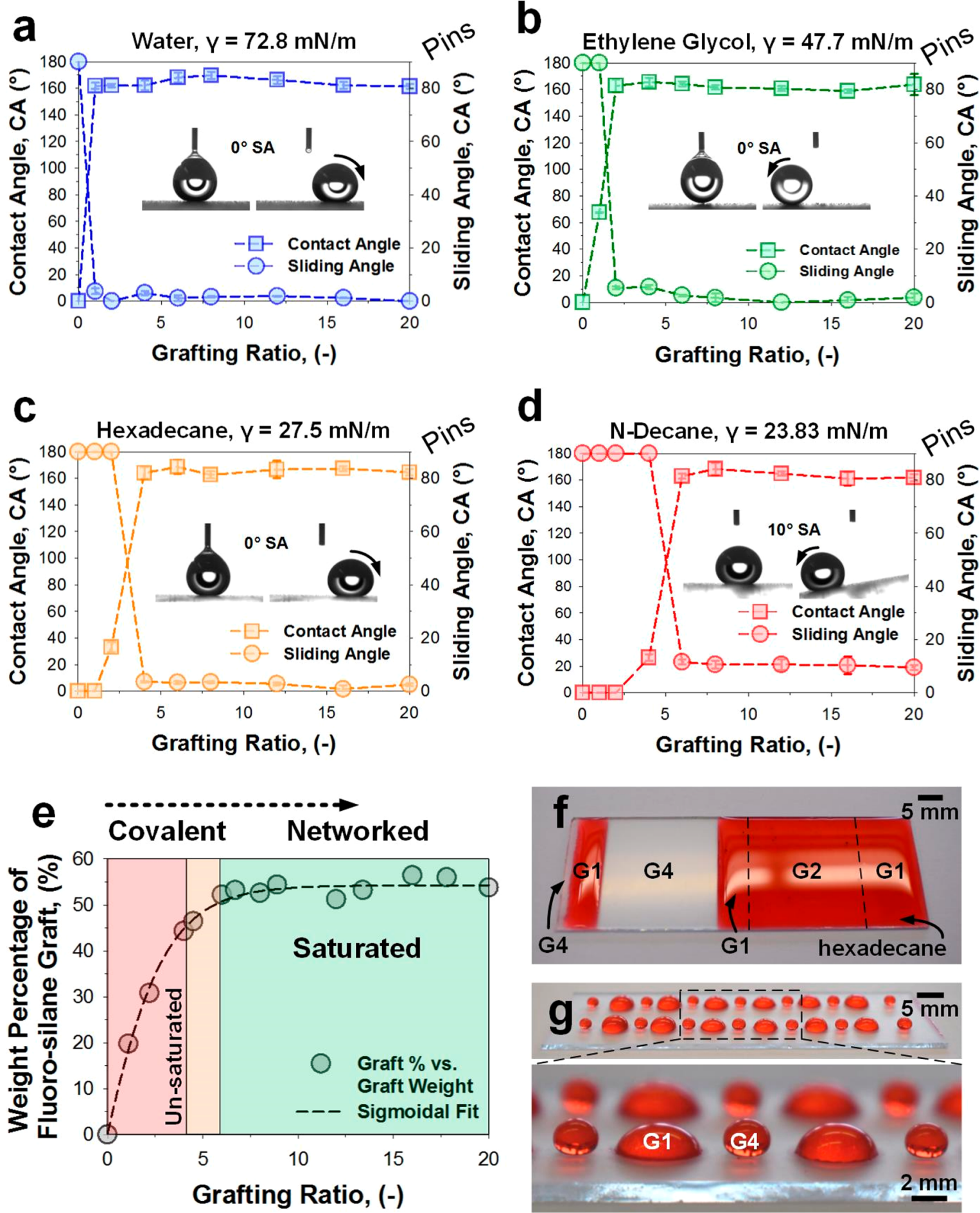

Figure 2. Progressive surface functionalization regimes vs wetting properties. Cassie-Baxter states $\left(\mathrm{CA}>150^{\circ}, \mathrm{SA}<10^{\circ}\right)$ against various liquids were achieved at respective reaction grafting ratios to surface tensions: (a) $1: 1,72.8 \mathrm{mN} / \mathrm{m}$, (b) $2: 1,47.7 \mathrm{mN} / \mathrm{m},(\mathrm{c}) 4: 1,27.5 \mathrm{mN} / \mathrm{m}$, and (d) $6: 1$, $23.83 \mathrm{mN} / \mathrm{m}$. (e) Grafting density of nanoparticles was ranged using the as-defined reaction grafting ratio (micromole per square meter) from 0 to 20 times. A range of weight percentage grafting was achieved from 0 to ca. 54\%, respectively. The tunable super-hydrophobic-super-oleophobic properties of covalent-network functionalized silica demonstrated in (f) linear and (g) radial droplet capturing and patterning. Liquid sheets and droplets are selectively captured by the one-step deposition of higher surface energy domains (super-hydrophobic but not super-oleophobic). The liquid capture systems in panels $\mathrm{f}$ and $\mathrm{g}$ were tuned to water and hexadecane but are, in principle, applicable to a range of other liquids.

vertical network polymerization. ${ }^{44}$ However, it has never been used in tuning surface densification of functional groups.

In this work, reaction graft ratio refers to the reaction formulation, indicated by the ratio of trichlorosilane used per surface area: $\mu \mathrm{mol} . \mathrm{m}^{-2}$. Here, the reaction graft ratio is also represented under the acronym G1-20, representing a ratio of $1: 1$ to $20: 1$, respectively. For example, the reaction grafting ratio of 16:1 (or G16) would refer to using $16 \mu$ moles of trichlorosilane per square meter of silica. Grafting density refers to the actual successful organic grafting after 

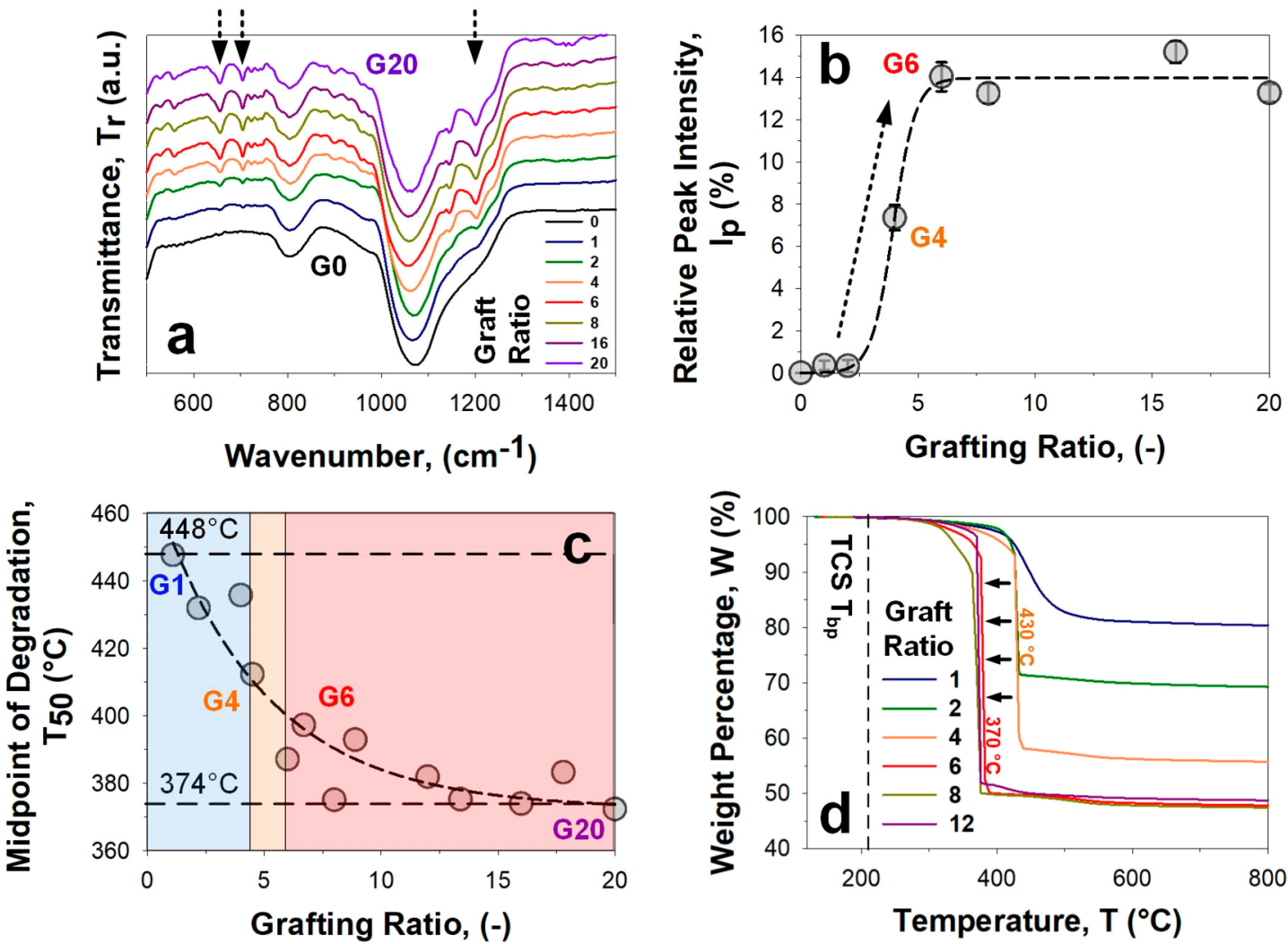

Figure 3. Thermogravimetric behavior and surface functionalization regimes. (a) Increasing magnitude of $720-725 \mathrm{~cm}^{-1}$ and 1147,1248 , and $1350 \mathrm{~cm}^{-1}$ peaks are indicative of increasing $\mathrm{C}-\mathrm{H}_{2}{ }^{65}$ and $\mathrm{C}_{-} \mathrm{F}_{2}{ }^{66}$ groups, respectively. This is interpreted as an enhanced surface density of fluorobased moieties on nanoparticles. (b) Relative peak intensity (normalized) at 655 and $704 \mathrm{~cm}^{-1}$ with respect to a control sample. (c) The $T_{-50}$ (midpoint of degradation) revealed a gradual drop $\left(\Delta T \approx 74{ }^{\circ} \mathrm{C}\right.$ ), contrasting $T_{\mathrm{d}-\text { onset }}$ (onset of decomposition). This decreased from a reaction grafting ratio of $1: 1$ with $448{ }^{\circ} \mathrm{C}$ down to $374{ }^{\circ} \mathrm{C}$ at reaction grafting ratios of $6: 1$ onward to $20: 1$ with a range of ca. $\pm 15{ }^{\circ} \mathrm{C}$ in variations. $(\mathrm{d})$ Thermogravimetric analysis of $T_{\mathrm{d}-\text { onset }}$ revealed consistent drops from ca. $430{ }^{\circ} \mathrm{C}$ across reaction graft ratios of $1: 1$ to $4: 1$. A sharp shift to $370{ }^{\circ} \mathrm{C}$ $\left(\Delta T \approx 60^{\circ} \mathrm{C}\right)$ was observed when increasing the reaction graft ratio beyond $6: 1$ and was tested up to $12: 1$ with highly consistent behaviors. Weight percentage losses at maximum thermal decomposition are ca. 20\%, 31\%, 44\%, and, finally, 54\% w/w at 1:1, 2:1, 4:1, and 6:1 (and beyond), respectively.

functionalization, represented as a $\mathrm{w} / \mathrm{w} \%$, bonded silane chains per square nanometer or micromoles per square meter. Both graft ratio and grafting density are represented in micromoles per square meter for consistency. For instance, a 6:1 reaction grafting ratio (reaction silane content of $6 \mu \mathrm{mol}$. $\mathrm{m}^{-2}$ ) will only result in a $4 \mu \mathrm{mol} \cdot \mathrm{m}^{-2}$ resultant grafting density. It thus facilitates an understanding of grafting efficiency, i.e., 4/ $6=67 \%$. Additional silane from 6:1 (i.e., 8:1 to 20:1) does not result in greater resultant grafting density, thus achieving supersaturation of the silica surface. Grafting density was determined by thermogravimetric analysis. As fumed silica does not decompose during thermal treatment, loss in weight during thermogravimetric tests enables direct computation of grafting density (see the Supporting Information).

A single and well-investigated source of fumed silica was used, with a Brunauer-Emmett-Teller (BET) surface area of ca. $395 \mathrm{~m}^{2} \cdot \mathrm{g}^{-1}$. Nanoparticles of different roughness tend to be synthesized from different methods, giving rise to variable surface hydroxyl group densities. ${ }^{39}$ Such known variations prompted the focus on a single source of material while varying functionalization extents to preserve a quasi-univariate comparison (surface functionalization vs wettability). Results indicate the densification of fluoralkyl groups on fumed silica nanoparticles, which was observed to result in improved superlyophobic properties, as illustrated in Figure 1b. This process enabled much-higher functionalization chain density than that previously reported by Campos et al. on fumed silica: 0.9 silane chains per square nanometer or just $27.3 \% \mathrm{w} / \mathrm{w}$, even with a similar fluoroalkyl-functional monochlorosilane. ${ }^{39}$ In contrast to Campos' monolayer functionalization design (Figure 1a, covalent), our multilayer covalent-oligomeric network system (Figure 1a, network) demonstrated an average maximum grafting density of ca. $53.6 \% \mathrm{w} / \mathrm{w}$, at 2.3 silane chains per square nanometer. The grafting density of silane chains per square nanometer is calculated based on thermogravimetric analysis, via methods proposed by Campos et al. ${ }^{39}$ Details are provided in eqs 1 and 2 in the Supporting Information. The grafted network system is also highly stable: Nanoparticles are typically washed in a centrifuge for multiple cycles (chloroform), dried in heated ovens, and finally ultrasonically dispersed at elevated temperatures in acetone $\left(\sim 80{ }^{\circ} \mathrm{C}\right)$ (see the Supporting Information).

This work highlights the unconventional use of a trichlorosilane in achieving ultra-densified surface functionalization (Figure 1c), potentially representing a new class of methods for realizing multilayered surface grafting. The corresponding functionalization-functionality regimes associated with maintaining the Cassie-Baxter state with liquids of distinctively demarcated surface tensions are presented in Figure S2. To this end, we will also present these surface- 


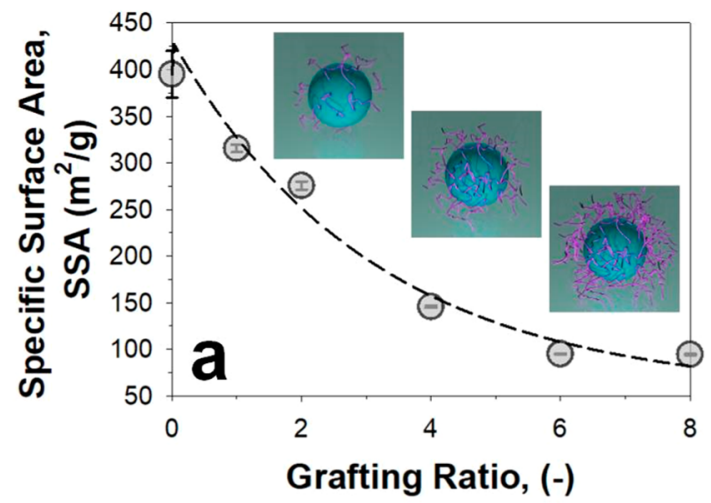

\section{b Covalent Grafting Network Grafting}
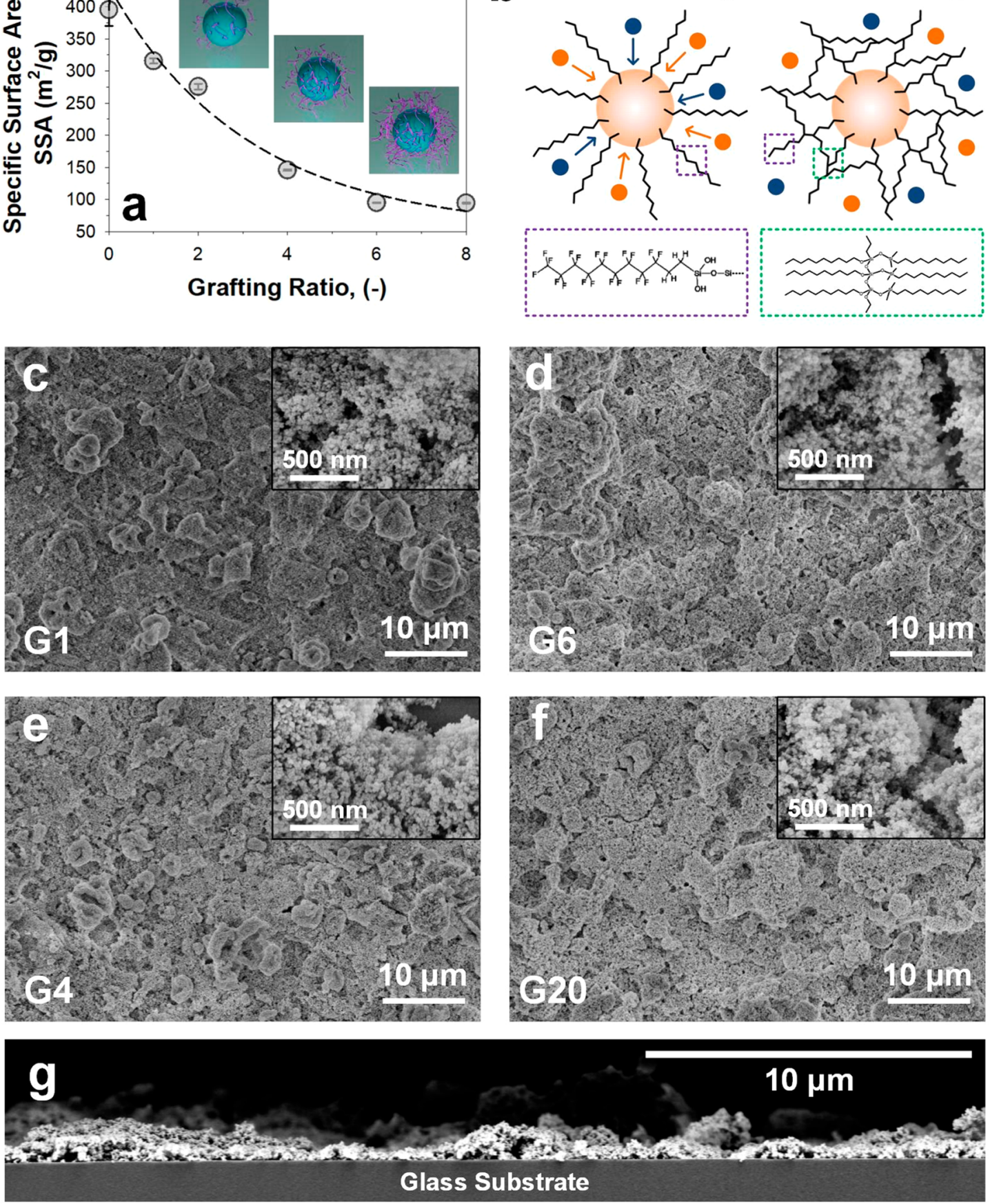

Figure 4. Macro-, micro-, and nanomorphology of network-functionalized silica nanoparticles. (a) Decrease in the effective specific surface area of fluoro-functionalized nanoparticles indicative of a dense networked layer (insets). Gradual decrease from ca. 400 to $100 \mathrm{~m}^{2} / \mathrm{g}$. This effect tapers off between 6:1 to 8:1 reaction graft ratios, indicative of surface saturation. (b) Monolayer covalently functionalized nanoparticles without $-\mathrm{OH}$ shielding and a covalent-oligomeric network-functionalized nanoparticle with $-\mathrm{OH}$ shielding. Water and oil entities are labeled with blue and orange spheres, respectively. Compositions of (purple) straight-chain covalent attachments and (green) oligomeric fluoro-silica networks. $\mathrm{F}$ and $\mathrm{H}$ atoms are not illustrated for conciseness. Scanning electron microscopy of nanoparticulate surfaces with reaction graft ratios of (c) 1:1, (d) 4:1, (e) 6:1, and (f) 20:1. Morphologies are hierarchical but near-identical, with microglobular features composed of nanospherical particles. (g) Side profile of a coated surface (G6 on glass). Glass substrate contrasted for reference. Additional high-magnification and side-profile geometries are included in Figure S10 for reference.

chemistry tunable super-hydrophobic to super-amphiphobic systems against a range of highly wetting synthetic and natural liquids.

Effect of Supersaturation Functionalization on Wettability. The grafting density of fluorosilane-functionalized silica is first correlated to wettability by direct wetting analysis. The $1: 1$ reaction graft ratio resulted in $20 \% \mathrm{w} / \mathrm{w}$ organic content, which is functional with water, achieving super-hydrophobicity. However, it remains wettable (Figure $2 \mathrm{a}$ ) by lower surface tension liquids such as ethylene glycol $(\gamma=47.7 \mathrm{mN} / \mathrm{m})$. A 
step increment at 2:1 reaction graft ratio gave rise to $30 \% \mathrm{w} / \mathrm{w}$ organic content, which is super-lyophobic to ethylene glycol but is wettable (Figure $2 \mathrm{~b}$ ) by hexadecane $(\gamma=27.5 \mathrm{mN} / \mathrm{m})$. A $4: 1$ reaction graft ratio reached up to $45 \% \mathrm{w} / \mathrm{w}$ organic content, which is super-oleo(amphi)phobic functional with hexadecane but remains wettable (Figure 2c) by liquids of even lower surface tensions, such as $n$-decane $(\gamma=23.8 \mathrm{mN}$ / $\mathrm{m})$. A final equilibrium reaction graft ratio of $6: 1$, representing ca. $52 \% \mathrm{w} / \mathrm{w}$ organic content is super-oleo(amphi)phobic functional (Figure 2d), even with $n$-decane, but fails (see Figure S3) with a lower surface tension liquid such as $n$-octane $(\gamma=21.62 \mathrm{mN} / \mathrm{m})$. A super-oleophilic contact angle of $0^{\circ}$ was noted up to G4, before ca. $50-90^{\circ}$ between G6 to G20. This equilibrium plateau persists even with further increments in reaction grafting ratio, tested up to a ratio of 20:1.

Wetting analysis was then supplemented by thermogravimetrically determining weight percent of the organic graft (Figure 2a). Experiments indicate the supersaturation of grafted organic chains at up to ca. $54 \% \mathrm{w} / \mathrm{w}$, corresponding to ca. $4 \mu \mathrm{mol} \cdot \mathrm{m}^{-2}$ (Figure $2 \mathrm{e}$, green regions). The sub- and supersaturation of fluoroalkyl density is thus correlated to various states of enhanced super-dewettability.

To visually exemplify these variances in wetting properties, contrasting super-hydrophobic (covalent functionalized) to super-oleophobic (network functionalized) surfaces were used in immersion-aided linear (Figure 2f) and droplet liquid patterning (Figure $2 \mathrm{~g}$ ). The rapid one-step deposition of higher surface energy domains allows facile creation of superhydrophobic-super-oleophilic sites (G1) on premade superamphiphobic (G4) substrates. Thereafter, immersion within water does not permit liquid capture, but immersion within hexadecane will rapidly result in captured liquid patterns (see Movies M1 and M2). Selective lyophilicity is adjustable simply by depositing specifically tuned nanoparticulate broths (G1G6). Patterning resolution, demonstrated here at ca. $1 \mathrm{~mm}$, is, in principle, only limited by the dimensions of the masks.

Spectroscopic and Thermogravimetric Analysis of Supersaturation Functionalization. The grafting dynamics were further analyzed by Fourier transform infrared (FTIR) spectroscopy and TGA. FTIR spectra revealed increasingly strong signals between 500 to $800 \mathrm{~cm}^{-1}$, corresponding to the increasing reaction graft ratios of silanes used. These signals confirm the presence of $-\mathrm{CF}_{2}$ and $-\mathrm{CF}_{3}$ groups (Figure $3 \mathrm{a}$ ). Quantitative analysis of maximum peak intensity at 655 and $704 \mathrm{~cm}^{-1}$ revealed a sigmoidal trend, with slow initial increments of $+0.526 \%$ and $+0.540 \%$ at $1: 1$ and $2: 1$ ratios, respectively (Figure 3b). At 4:1 and 6:1 ratios, the peak intensity spikes and reaches $+7.78 \%$ and $+14.5 \%$, respectively. Further increments in reaction grafting ratio (8:1 to 20:1) led to a plateaued peak intensity of between 13 and $15 \%$ (Figure $3 \mathrm{~b})$. This step-increase at $4: 1$ is indicative of the accelerated network polymerization reaction, which results in the densified 3D fluoroalkyl layer. The supersaturation functionalization achieves a quantitative maximum at 6:1 reaction grafting ratio, in line with previous wetting and subsequent thermogravimetric data.

The $T_{-50}$, or the midpoint of thermal degradation was then analyzed for the series of sub- and supersaturation-functionalized nanoparticles. The behavior of $T_{-50}$ was notably smooth and exponential, without distinctive step-like behaviors between reaction grafting ratios of $1: 1$ to $6: 1$. A maximum $T_{-50}$ of $448{ }^{\circ} \mathrm{C}$ at a reaction grafting ratio of $1: 1$ decreased to an equilibrium $T_{-50}$ of $382 \pm 9{ }^{\circ} \mathrm{C}$ at equilibrium ratios of $6: 1$ to 20:1 (Figure 3c, red domain). This occurs counterintuitively to the overall thermogravimetric weight analysis, in which an apparent step-shift occurs between the reaction grafting ratios of $4: 1$ to $6: 1$, from ca. 430 to $370{ }^{\circ} \mathrm{C}$ (Figure 3d, orange to red lines). This step-shift behavior mirrors the FTIR peak analysis.

These step-shifts (TGA and FTIR) suggest that the increase in final grafting density occurs within a predominantly stoichiometric regime via standard covalent bonding up to a reaction grafting ratio of $4: 1$. Thereafter, at and beyond a ratio of $6: 1$, a transitional regime involving both covalent and network polymerization (horizontal and vertical) dominates. ${ }^{44}$ This enables multilayered, multisite configurations that involve the stacking of multiple fluoroalkyl chains, thus further decreasing net surface energy density. However, owing to the smooth exponential decay behaviors in $T_{-50}$ (Figure $3 \mathrm{c}$ ), such multisite stacking must have also occurred spontaneously during initial covalent bonding, i.e., 1:1 or 2:1 ratios, albeit to a much-lesser extent.

The multilayered oligomeric stacking mechanism is proposed alongside network packing of $\mathrm{Si}-\mathrm{O}$ bonds. In the monolayer design, the formation of $3 \mathrm{Si}-\mathrm{O}$ bonds with the substrate is usually not possible due to steric hindrance. This results in excess hydroxyl groups and a relatively relaxed $\mathrm{Si}-$ $\mathrm{O}-\mathrm{Si}$ tetrahedral configuration (free rotation) that is tightly bound to the interface (Figure 1a, covalent). However, during the oligomeric formation of the poly fluoro-network, the $\mathrm{Si}-$ $\mathrm{O}-\mathrm{Si}$ bonds are formed in a dominantly vertical ${ }^{44}$ tetrahedral configuration. These $\mathrm{Si}-\mathrm{O}$ bonds (within the $-\mathrm{Si}-\mathrm{O}-\mathrm{Si}-$ backbone) exist within a network and experience severe bond strain due to steric hindrance from large fluoroalkyl groups (Figure 1a, network). As a result, they are oligomeric in nature and behave more like polysiloxane ${ }^{46}$ compounds instead of inorganic silica. Thus, during thermogravimetric analysis, these relatively less-stable oligomeric polysiloxane backbones undergo $\mathrm{Si}-\mathrm{O}$ scission, ${ }^{47}$ resulting in the large scale disassembly of the fluoro-silica network. The breakdown of this network occurs at markedly lower temperatures compared with purely covalent but singularly bonded monolayer configurations. However, some covalent bonding must still be present because weight loss and thermal decomposition only occur upwards of $>300{ }^{\circ} \mathrm{C}$, with a minimum $T_{50}$ (midpoint of degradation) of $374{ }^{\circ} \mathrm{C}$ (Figure $3 \mathrm{~d}$ ). If no covalent bonding remains, thermal evaporation of silanes should have taken place at $\leq 216{ }^{\circ} \mathrm{C}$ (boiling point of heptadecafluoro-1,1,2,2-tetrahydrodecyl trichlorosilane).

These results are supported by the specific surface area (SSA) analysis using the Brunauer-Emmett-Teller (BET) technique (Figure $4 \mathrm{a}$ ). SSAs of functionalized nanoparticles were found to exponentially decrease from ca. $395 \mathrm{~m}^{2} / \mathrm{g}$ (ungrafted), to a plateau of ca. $94 \mathrm{~m}^{2} / \mathrm{g}$ (6:1 to $8: 1$ supersaturation ratios). The multilayer stacking of functional fluoroalkyl chains forms a shell-like network around the nanoparticles, resulting in decreased surface areas. This decrease is unprecedentedly lower than that previously reported by the most-optimized monolayer functionalization of fumed silica (Campos et al., $184 \mathrm{~m}^{2} / \mathrm{g}$ ). ${ }^{39}$ This is strongly suggestive of the supersaturation functionalization mechanism. In this work, results from thermogravimetric and specific surface area analysis (Figures 3-4) support the hypothesis of gradually decreasing effective surface area with increased grafting ratios. This event occurred up to the point of supersaturation via network-functionalization mechanisms. ${ }^{44}$ 


\section{$-0.17 \mathrm{~ms} \quad 0 \mathrm{~ms}$ \\ $1.50 \mathrm{~ms}$ \\ $2.67 \mathrm{~ms}$ \\ Water, $72.8 \mathrm{mN} / \mathrm{m}$}

$6.67 \mathrm{~ms}$

$12.33 \mathrm{~ms}$

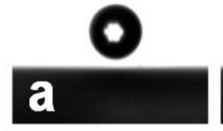

$-0.17 \mathrm{~ms}$

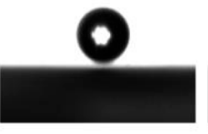

$0 \mathrm{~ms}$

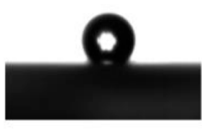

$0.33 \mathrm{~ms}$ Ethylene

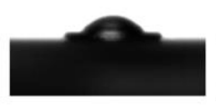

$1.50 \mathrm{~ms}$

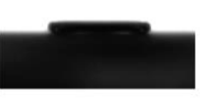

$2.67 \mathrm{~ms}$ $7.7 \mathrm{mN} / \mathrm{m}$
$6.67 \mathrm{~ms}$
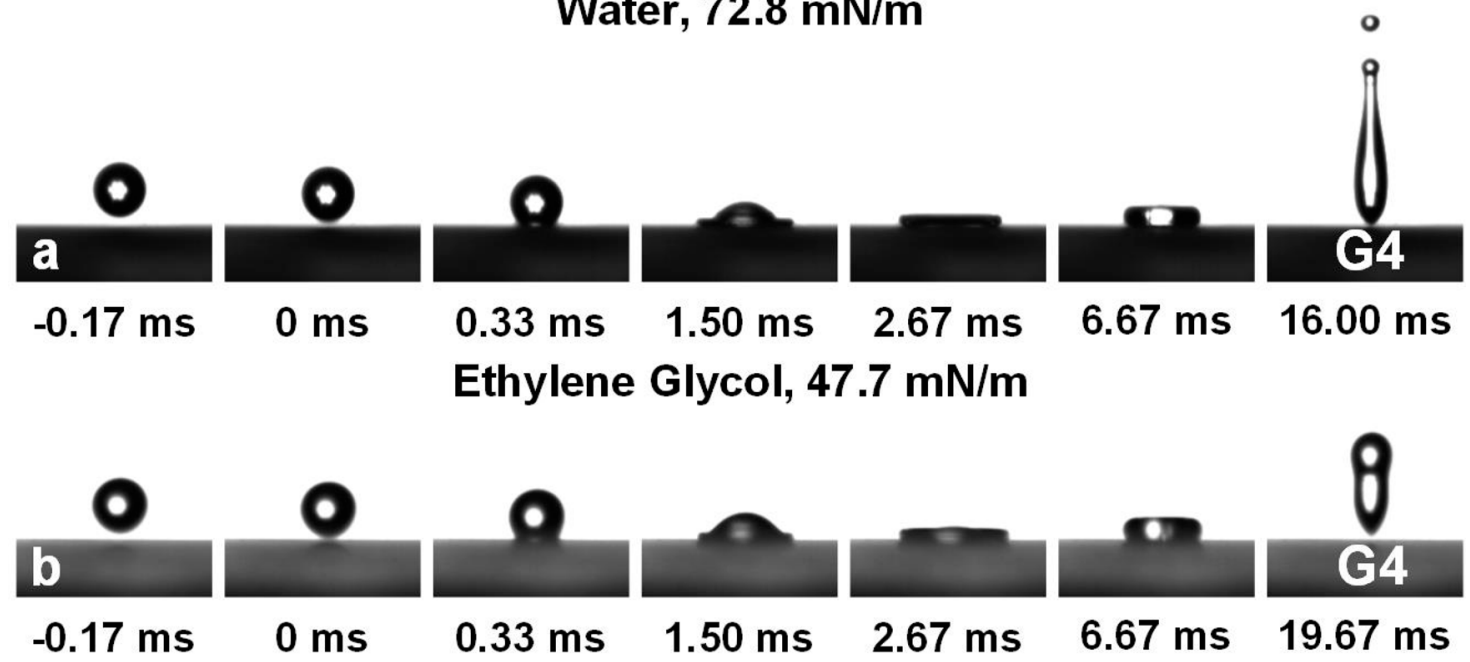

\section{Hexadecane, $27.47 \mathrm{mN} / \mathrm{m}$}
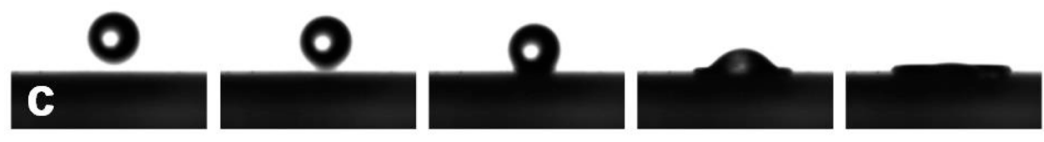

$6.67 \mathrm{~ms}$

$19.67 \mathrm{~ms}$
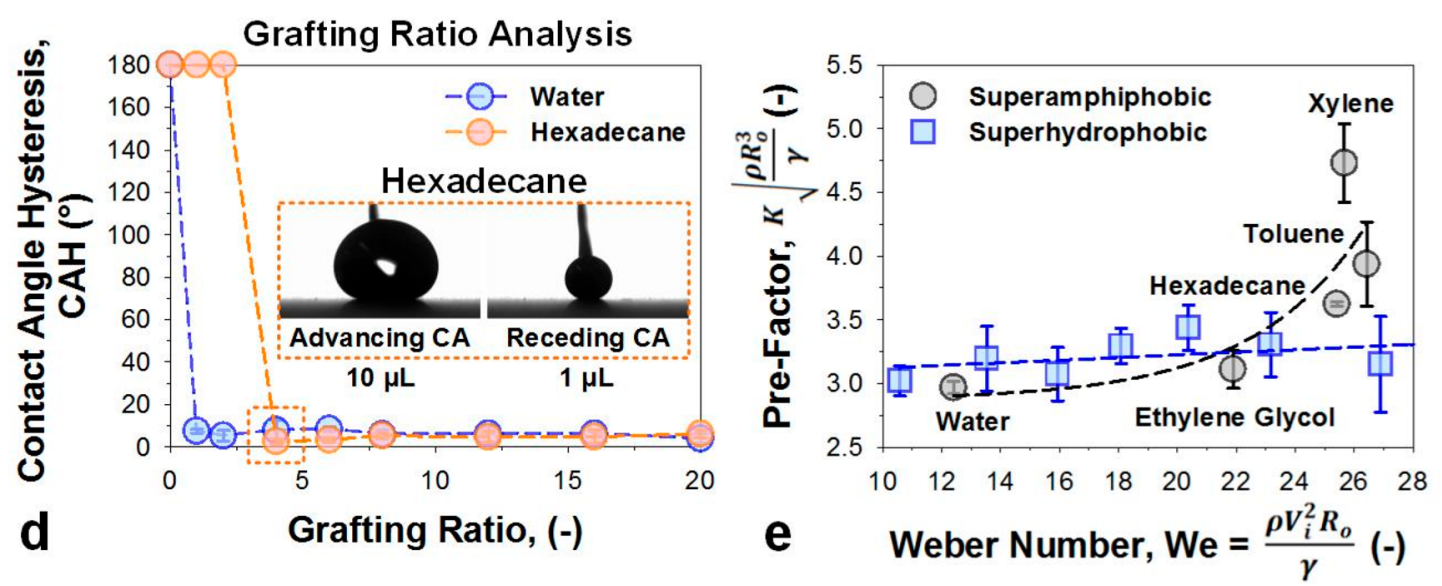

Figure 5. Super-amphiphobicity assessed under impact studies. Droplet impact analysis on the as-developed G4 (reaction grafting ratio of 4:1) super-amphiphobic coatings, showcasing clean droplet impact and detachment from (a) water, (b) ethylene glycol, and (c) hexadecane. (d) Contact-angle hysteresis measurements (G1 to G20, reaction grafting ratios of 1:1 to 20:1) by using a hexadecane probe liquid $(\gamma=27.5 \mathrm{mN} / \mathrm{m}$ ). A measurement of $180^{\circ}$ indicates super-lyophilic properties, while ultra-low CAH values were obtained at and beyond a reaction grafting ratio of 4:1. Stable wetting behavior is observed throughout the entire advancing-receding cycle for all surfaces. (e) Analysis of the capillary inertial dynamics via the pre-factor of G4 (reaction grafting ratio of 4:1). The pre-factor under super-hydrophobic impact is consistent (ca. 3-3.5) and can be scaled by a linear line. The pre-factor under super-amphiphobic impact is scaled with an exponential curve, with sharp increments in magnitude under the use of low-surface-tension liquids such as hexadecane, toluene, and xylene.

In previous studies involving nanoparticles or nanofilaments, ${ }^{22,30}$ reports often always attribute the super-amphiphobic effects achieved to the presence of stochastic re-entrant geometries. For instance, Seeger ${ }^{30}$ and Vollmer ${ }^{22}$ linked superamphiphobicity to the use of re-entrant nanofilaments ${ }^{30}$ and nanospheres, ${ }^{22}$ respectively. The resulting spherical side profiles are well-documented for their re-entrant properties. ${ }^{33}$ In contrast to these works, the proposed method uses fumed silica, which has the morphology of branched fractals. They scarcely represent the ideal geometry required. However, the use of increasing graft density demonstrates improving super liquid repellency, from super-(hydro-to-amphi)-phobicity on the wet-spray-coated, but unoptimized morphology through four sequential and distinct degrees of functionalization. Surface enhancement is key in this study, aiming to provide quantifiable links between super-liquid repellency and fluorofunctionalization density. This is, as of the time of writing, knowledge that has yet to be discussed in the literature.

The networked fluoroalkyl shell is believed to be capable of shielding $^{48}$ any remaining surface energetic hydroxyl $(-\mathrm{OH})$ groups that are typically unshielded in monolayer variants (Figure $4 b$ ), thus resulting in enhanced dewetting. This mode 
of multilayer supersaturation functionalization has never been previously proposed in the literature. Understanding and achieving this concept may generate insights toward new modes of enhanced surface modifications.

Surface Morphology and Optical Properties. Increased superlyophobic performance can often be traced toward enhancements in surface morphologies..$^{24,32,49-51}$ Here, surface morphological variations were minimized owing to the method of synthesis. All surfaces were fabricated by wet-spray deposition. Functionalized nanoparticles were first dispersed in acetone $(10 \mathrm{mg} / \mathrm{mL})$. The colloidal solution is highly stabilized. This solution is then spray-deposited onto substrates as partially wet films at 3 bar of pressure from a distance of 10 $\mathrm{cm}$. Qualitative micro- and nanoscale analysis of these stochastically developed nanoparticle agglomerates appear to be nearly identical regardless of reaction grafting ratio and resultant graft density (Figures $4 \mathrm{c}-\mathrm{f}$ and S12). To this end, specific experimental design parameters were employed: (1) use of a consistent type of silica material with well-investigated surface properties ${ }^{39}$ (all surface functionalization thus builds upon an identical skeletal base); (2) use of identical reaction conditions with univariately increased surface functionalization; and (3) stochastically controlled spray-deposition under identical (a) colloidal concentration and solvent volatility, ${ }^{52-54}$ (b) spray parameters (mechanical), ${ }^{55}$ and (c) ambient temperature. ${ }^{56}$ Current literature suggests negligible macroscopic structural variations during wet-spray deposition under such identical deposition parameters. ${ }^{27,57,58}$ In addition, considering that the range of surface functionalization is almost identical in nature (fluoroalkyl moieties), it is unlikely that macrostructural differences are possible between variants (Figure 4). ${ }^{59}$ Surfaces developed are all composed of micronsized globules with nanoscale roughness, thus possessing surface hierarchy typical of nanoparticle coatings. Side-profile analysis supports these top-down observations (Figures $4 \mathrm{~g}$ and S12). These surfaces reflect the classical capillary-contracted nanoparticle-based film (Figure $4 \mathrm{c}-\mathrm{f}$ ).

This is further supported by scanning electron micrographs that highlights microscopic features found on the sideprofiles (Figure S10), which do not reveal distinguishable characteristics between the variants. All results are indicative of identical nano-, micro-, and macro- morphologies across different grafting densities. Optical behaviors of the films and corresponding details regarding inherent material property variations pre- and post-functionalization area also included in the Supporting Information and Figure S4 for reference. Optical data were presented for scientific clarity; however, measurements may have been inherently limited by the mode of synthesis and characterization at G6 and beyond (see the Supporting Information).

Droplet Impact and Liquid Stability. These supersaturation fluoro-functionalized silica coatings were hereafter subjected to droplet-impact tests. This was assessed ${ }^{60}$ via a $5 \mu \mathrm{L}$ droplet of hexadecane at $0.94 \mathrm{~ms}^{-1}$. Surprisingly, optimal superamphiphobic variants including those between 6:1 to 20:1 reaction grafting ratios appear to exhibit different dropletimpact stability, often encountering Wenzel penetration (Figure S5). This is currently attributed to the significantly altered surface affinity between the densely functionalized fluoroalkyl-silica (G6). During droplet impact, immense stress is placed upon the macro-nanoparticle-assembly. Coupling nanostructural configurations with extremely low surface energy density could result in mechanical instabilities between nanoparticles. Local disintegration of the interface may thus induce droplet penetration and pinning.

Droplet-impact studies were later focused on the most-stable super-amphiphobic variant, synthesized under a reaction grafting ratio of $4: 1$. This configuration demonstrates excellent stability against a variety of high-to-low surface tension liquids, such as water, ethylene glycol, xylene, toluene, and hexadecane (Figures $5 \mathrm{a}-\mathrm{c}$ and S6). These results are first supplemented by contact-angle hysteresis ( $\mathrm{CAH})$ measurements, which were performed via a standard probe liquid, hexadecane (Figure 5d). ${ }^{51,61}$

During droplet impact, clear transitional impact-wetting properties were exhibited. This is useful for assessing the dynamic wetting stability of such supersaturation functionalized nanoparticulate interfaces. This was subsequently assessed via the use of a variety of liquids, which results in a tunable range of Weber numbers. Later, it was represented against the pre-factor of the inertial capillary time scale. Most notably, it showcased very different impact dynamics compared with traditional re-entrant-based nanoagglomerate interfaces. $^{29}$

In previous studies, re-entrant particle agglomerate interfaces typically experience consistent pre-factors over a wide range of Weber numbers (Figure S7). ${ }^{29}$ Here, super-hydrophobic droplet impact properties assessed using water appears to have a gradually appreciating but stable prefactor (Figures 5e and S8). However, transitional behaviors were revealed with the use of low surface tension liquids, revealing a rise in the pre-factors between the use of ethylene glycol and hexadecane. Despite clean detachment for all liquids tested in this range, the increasing pre-factors are indicative of different capillary infiltration-exfiltration dynamics. Notwithstanding these extreme impact conditions, these nanoparticle-coated surfaces remain highly stable and functional during conventional usage (Movie M3 and Figures S9 and S11). Nanoparticle coatings developed by wet-spray deposition tend to be liquid-stable, owing to the strong capillary pull that assembled the microand nano- structural morphology. ${ }^{62}$ This effect is fairly welldemonstrated in various research works, in which the crosslinking of individual particles is not necessary for liquid stability. ${ }^{63,64}$ They are also further tested against a series of real-world liquids (Movie M3) and even food surfactant (glycol proteins) infused drops. While the nanoparticle coatings can withstand multiple droplet impacts (100 droplets, $\left.\sim 0.94 \mathrm{~ms}^{-1}\right)$, jet streams, and immersion (1-2 cm of solvent, i.e., hexadecane, $30 \mathrm{~s}$ ), they are not abrasion-stable. Notably, the derivation of super-amphiphobicity is independent from precisely engineering re-entrant geometries. These chemically tunable super-amphiphobic nanoparticles can thus be eventually incorporated into systems of binders or macroscopic structures for enhanced durability. ${ }^{5,6,31}$ Future work will involve incorporating a binder system to improve abrasionbased mechanical stability.

Conclusions. In this work, we demonstrate a quantifiable link between super liquid-repellency and fluoro-functionalization density. To achieve this, we introduced a new concept in enabling the step-wise densification of surface functionalization, termed supersaturation functionalization. This was achieved by facilitating the controlled formation of a multilayer, self-condensing fluoroalkyl network around the target nanoparticle. The technique resulted in tunable grafting density, reaching a maximum of $54 \% \mathrm{w} / \mathrm{w}$. More importantly, these surface-enhanced materials showcased unprecedented 
step-wise variations in super-liquid repellency, achieved simply through functionalization densification. The effect of grafting density was thoroughly investigated within the domains of super-hydrophobicity and super-oleophobicity and, for the first time, correlated the density of grafted fluoroalkyl moieties to effective wettability. The maximum densification achieved a Cassie-Baxter state even with surface tensions down to 23.8 $\mathrm{mN} / \mathrm{m}$. These findings are instrumental toward potential routes that reduce the need for stringent surface morphological requirements, such as highly precise re-entrant geometries for achieving super-oleo(amphi- or omni-)phobicity. It highlights new knowledge that even with non-optimal surface morphologies, super-hydro(amphi)phobicity can be tuned and improved with the right surface chemistry. We demonstrate here, a process that is facile, scalable, and highly tunable. It can also be of immediate impact to various research and industrial sectors requiring the high-density loading of functional surface groups. In the future, we hope that the techniques and concepts demonstrated here can extend beyond the fields of wettability, perhaps taking root in biotechnology, nanorobotics, nanobiocatalysis, and clean-energy research.

\section{ASSOCIATED CONTENT}

\section{S Supporting Information}

The Supporting Information is available free of charge on the ACS Publications website at DOI: 10.1021/acs.nanolett.8b04972.

Additional details on experimental materials and methods; figures showing proposed mechanisms for super-saturation functionalization, a functionalization roadmap, wetting properties, white-light interferometric studies, droplet-impact studies, pre-factor analysis, droplet patterning, supporting SEM analysis (side profile and high magnifications), and super-liquid repellency against real-world and synthetic liquids (PDF)

A video showing linear patterning (AVI)

A video showing droplet patterning (AVI)

A video showing a coated cardboard substrate (AVI)

\section{AUTHOR INFORMATION}

\section{Corresponding Author}

*E-mail: william.wong@anu.edu.au and wong@mpip-mainz. mpg.de.

\section{ORCID $\odot$}

William S. Y. Wong: 0000-0002-5389-5018

\section{Notes}

The author declares no competing financial interest.

\section{ACKNOWLEDGMENTS}

W.S.Y.W acknowledges the $\mathrm{PhD}$ research fellowship from the Australian National University and the Marie SkłodowskaCurie ESR fellowship under the Horizon 2020 program (LubISS network grant agreement no. 722497). W.S.Y.W. thanks A. Naga, M. Mueller, D. M. Garcia-Gonzalez, Prof. Doris Vollmer, and Prof. Hans Juergen Butt from the Physics of Interfaces group at the Max Planck Institute for Polymer Research for their insight. Funding from the Discovery Translation Fund (DTF238) at the ANU is gratefully acknowledged. The use of laboratories belonging to Prof. Vincent Craig (RSPE, ANU) and A/Prof. Antonio Tricoli (RSE, ANU) is appreciated. W.S.Y.W. thanks X. Gao (RSE,
ANU) for his assistance during the scanning electron microscopy analysis. Access to the facilities of the Centre for Advanced Microscopy (CAM) with funding through the Australian Microscopy and Microanalysis Research Facility (AMMRF) is gratefully acknowledged. This work was conceptualized at the Australian National University and completed at the Max Planck Institute of Polymer Research.

\section{REFERENCES}

(1) Liu, M.; Wang, S.; Jiang, L. Nat. Rev. Mater. 2017, 2, 17036.

(2) Li, Y.; Chen, S.; Wu, M.; Sun, J. Adv. Mater. 2014, 26, 33443348.

(3) Zhou, H.; Wang, H.; Niu, H.; Gestos, A.; Wang, X.; Lin, T. Adv. Mater. 2012, 24, 2409-12.

(4) Deng, X.; Mammen, L.; Zhao, Y.; Lellig, P.; Mullen, K.; Li, C.; Butt, H. J.; Vollmer, D. Adv. Mater. 2011, 23, 2962-5.

(5) Lu, Y.; Sathasivam, S.; Song, J.; Crick, C. R.; Carmalt, C. J.; Parkin, I. P. Science 2015, 347, 1132-1135.

(6) Peng, C.; Chen, Z.; Tiwari, M. K. Nat. Mater. 2018, 17, 355360.

(7) Zhan, K.; Hou, X. Small 2018, 14, 1703283.

(8) Mazutis, L.; Gilbert, J.; Ung, W. L.; Weitz, D. A.; Griffiths, A. D.; Heyman, J. A. Nat. Protoc. 2013, 8, 870-891.

(9) Tian, J.; Kannangara, D.; Li, X.; Shen, W. Lab Chip 2010, 10, 2258-64.

(10) Wong, W. S. Y.; Liu, G.; Tricoli, A. Small 2017, 13, 1603688.

(11) Wong, W. S. Y.; Nasiri, N.; Liu, G.; Rumsey-Hill, N.; Craig, V. S. J.; Nisbet, D. R.; Tricoli, A. Adv. Mater. Interfaces 2015, 2, 1500071.

(12) Cho, S. J.; Nam, H.; Ryu, H.; Lim, G. Adv. Funct. Mater. 2013, 23, 5577-5584.

(13) Wu, D.; Wu, S. Z.; Chen, Q. D.; Zhang, Y. L.; Yao, J.; Yao, X.; Niu, L. G.; Wang, J. N.; Jiang, L.; Sun, H. B. Adv. Mater. 2011, 23, $545-9$.

(14) Tian, X.; Jin, H.; Sainio, J.; Ras, R. H. A.; Ikkala, O. Adv. Funct. Mater. 2014, 24, 6023-6028.

(15) Wong, W. S. Y.; Gengenbach, T.; Nguyen, H. T.; Gao, X.; Craig, V. S. J.; Tricoli, A. Adv. Funct. Mater. 2018, 28, 1704423.

(16) Li, C.; Li, N.; Zhang, X.; Dong, Z.; Chen, H.; Jiang, L. Angew. Chem., Int. Ed. 2016, 55, 14988-14992.

(17) Wong, W. S. Y.; Li, M.; Nisbet, D. R.; Craig, V. S. J.; Wang, Z.; Tricoli, A. Sci. Adv. 2016, 2, el600417.

(18) Py, C.; Reverdy, P.; Doppler, L.; Bico, J.; Roman, B.; Baroud, C. N. Phys. Rev. Lett. 2007, 98, 156103.

(19) Paulsen, J. D.; Demery, V.; Santangelo, C. D.; Russell, T. P.; Davidovitch, B.; Menon, N. Nat. Mater. 2015, 14, 1206.

(20) Ju, J.; Yao, X.; Hou, X.; Liu, Q.; Zhang, Y. S.; Khademhosseini, A. J. Mater. Chem. A 2017, 5, 16273-16280.

(21) Lafuma, A.; Quere, D. Nat. Mater. 2003, 2, 457-460.

(22) Deng, X.; Mammen, L.; Butt, H.-J.; Vollmer, D. Science 2012, $335,67-70$.

(23) Yong, J.; Chen, F.; Yang, Q.; Huo, J.; Hou, X. Chem. Soc. Rev. 2017, 46, 4168-4217.

(24) Liu, T. L.; Kim, C.-J. C. Science 2014, 346, 1096-1100.

(25) Im, M.; Im, H.; Lee, J.-H.; Yoon, J.-B.; Choi, Y.-K. Soft Matter 2010, 6, 1401-1404.

(26) Tuteja, A.; Choi, W.; Ma, M.; Mabry, J. M.; Mazzella, S. A.; Rutledge, G. C.; McKinley, G. H.; Cohen, R. E. Science 2007, 318, $1618-1622$.

(27) Vahabi, H.; Wang, W.; Movafaghi, S.; Kota, A. K. ACS Appl. Mater. Interfaces 2016, 8, 21962-21967.

(28) Rangel, T. C.; Michels, A. F.; Horowitz, F.; Weibel, D. E. Langmuir 2015, 31, 3465-3472.

(29) Wong, W. S. Y.; Liu, G.; Nasiri, N.; Hao, C.; Wang, Z.; Tricoli, A. ACS Nano 2017, 11, 587-596.

(30) Zhang, J.; Seeger, S. Angew. Chem., Int. Ed. 2011, 50, 6652-6.

(31) Wong, W. S. Y.; Stachurski, Z. H.; Nisbet, D. R.; Tricoli, A. ACS Appl. Mater. Interfaces 2016, 8, 13615-13623. 
(32) Tuteja, A.; Choi, W.; McKinley, G. H.; Cohen, R. E.; Rubner,

M. F. MRS Bull. 2008, 33, 752-758.

(33) Brown, P. S.; Bhushan, B. APL Mater. 2016, 4, 015703.

(34) Zhang, M.; Zhang, T.; Cui, T. Langmuir 2011, 27, 9295-9301.

(35) Kota, A. K.; Kwon, G.; Tuteja, A. NPG Asia Mater. 2014, 6, e109.

(36) Nishino, T.; Meguro, M.; Nakamae, K.; Matsushita, M.; Ueda, Y. Langmuir 1999, 15, 4321-4323.

(37) Wang, S.; Liu, K.; Yao, X.; Jiang, L. Chem. Rev. 2015, 115, $8230-8293$.

(38) Liu, K.; Cao, M.; Fujishima, A.; Jiang, L. Chem. Rev. 2014, 114, 10044-10094.

(39) Campos, R.; Guenthner, A. J.; Haddad, T. S.; Mabry, J. M. Langmuir 2011, 27, 10206-10215.

(40) Mabry, J. M.; Vij, A.; Iacono, S. T.; Viers, B. D. Angew. Chem., Int. Ed. 2008, 47, 4137-40.

(41) Kota, A. K.; Li, Y.; Mabry, J. M.; Tuteja, A. Adv. Mater. 2012, 24, 5838-5843.

(42) Pan, S.; Kota, A. K.; Mabry, J. M.; Tuteja, A. J. Am. Chem. Soc.

2013, 135, 578-581.

(43) Choi, W.; Tuteja, A.; Chhatre, S.; Mabry, J. M.; Cohen, R. E.; McKinley, G. H. Adv. Mater. 2009, 21, 2190-2195.

(44) Fadeev, A. Y.; McCarthy, T. J. Langmuir 2000, 16, 7268-7274.

(45) Berendsen, G. E.; Galan, L. d. J. Liq. Chromatogr. 1978, 1, 561586.

(46) Schiavon, M. A.; Redondo, S. U. A.; Pina, S. R. O.; Yoshida, I.

V. P. J. Non-Cryst. Solids 2002, 304, 92-100.

(47) Camino, G.; Lomakin, S. M.; Lazzari, M. Polymer 2001, 42, 2395-2402.

(48) Mammen, L.; Deng, X.; Untch, M.; Vijayshankar, D.; Papadopoulos, P.; Berger, R.; Riccardi, E.; Leroy, F.; Vollmer, D. Langmuir 2012, 28, 15005-15014.

(49) Wong, W. S. Y.; Gutruf, P.; Sriram, S.; Bhaskaran, M.; Wang,

Z.; Tricoli, A. Adv. Funct. Mater. 2016, 26, 399-407.

(50) Campos, R.; Guenthner, A. J.; Meuler, A. J.; Tuteja, A.; Cohen, R. E.; McKinley, G. H.; Haddad, T. S.; Mabry, J. M. Langmuir 2012, 28, 9834-9841.

(51) Zhao, H.; Law, K.-Y.; Sambhy, V. Langmuir 2011, 27, 59275935.

(52) Tsapis, N.; Dufresne, E. R.; Sinha, S. S.; Riera, C. S.; Hutchinson, J. W.; Mahadevan, L.; Weitz, D. A. Phys. Rev. Lett. 2005, 94, 018302.

(53) Sen, D.; Mazumder, S.; Melo, J. S.; Khan, A.; Bhattyacharya, S.; D'Souza, S. F. Langmuir 2009, 25, 6690-5.

(54) Tsapis, N.; Dufresne, E. R.; Sinha, S. S.; Riera, C. S.; Hutchinson, J. W.; Mahadevan, L.; Weitz, D. A. Phys. Rev. Lett. 2005, 94, 018302.

(55) Diao, Y.; Shaw, L.; Bao, Z.; Mannsfeld, S. C. B. Energy Environ. Sci. 2014, 7, 2145-2159.

(56) Su, B.; Caller-Guzman, H. A.; Korstgens, V.; Rui, Y.; Yao, Y.; Saxena, N.; Santoro, G.; Roth, S. V.; Muller-Buschbaum, P. ACS Appl. Mater. Interfaces 2017, 9, 43724-43732.

(57) Steele, A.; Bayer, I.; Loth, E. Nano Lett. 2009, 9, 501-505.

(58) Sasaki, K.; Tenjimbayashi, M.; Manabe, K.; Shiratori, S. ACS Appl. Mater. Interfaces 2016, 8, 651-9.

(59) Zellmer, S.; Lindenau, M.; Michel, S.; Garnweitner, G.; Schilde, C. J. Colloid Interface Sci. 2016, 464, 183-90.

(60) Richard, D.; Clanet, C.; Quere, D. Nature 2002, 417, 811-811.

(61) Wong, W. S. Y.; Tricoli, A. ACS Appl. Mater. Interfaces 2018, 10, 13999-14007.

(62) Ogihara, H.; Xie, J.; Okagaki, J.; Saji, T. Langmuir 2012, 28, 4605-4608.

(63) Ebert, D.; Bhushan, B. Langmuir 2012, 28, 11391-11399.

(64) Xu, L.; Karunakaran, R. G.; Guo, J.; Yang, S. ACS Appl. Mater. Interfaces 2012, 4, 1118-25.

(65) Jr, F. W. B. Textbook of Polymer Science; John Wiley \& Sons: Hoboken, NJ, 2007; p 578.

(66) Shaporenko, A.; Cyganik, P.; Buck, M.; Ulman, A.; Zharnikov, M. Langmuir 2005, 21, 8204-8213. 